\title{
La stazione sismica di Serra La Nave sull' Etna
}

\author{
A. Bottari - M. RiUSCetti (*)
}

Ricevuto il 10 Aprile 1967

Riassunto. - Si descrive la nuova stazione sismologica di Serra La Nave (Etna, Sicilia). Essa fa parte di una rete di stazioni in programma per la sorveglianza continua del vulcano.

Con riferimento a quanto si fa in questo campo in altri paesi, viene auspicato il potenziamento di questa e delle future stazioni mediante altri strumenti quali clinometri, gravimetri e magnetometri.

Summary. - This paper deals with the installation of the first seismological station on Mount Etna (Sicily). Three Willmore MK II short period seismometers with optical registration have been put into a little building, near Astrophysic Observatory at Serra La Nave, on the Southern side of the mountain. The coordinates of the station are: lat. $37^{\circ} 41^{\prime} 30^{\prime \prime} \mathrm{N}$, long. $14^{\circ} 45^{\prime} 22^{\prime \prime}, 9 \mathrm{E}$, height $1725 \mathrm{~m}$.

Four more stations will complete a net of seismological stations on Etna.

The characteristics of instruments are:

$$
\begin{aligned}
& \text { seismometers adjustable period from } 0,75 \text { to } 5 \mathrm{sec} \text { (horiz.) } \\
& \text { seismometers adjustable period from } 0,60 \text { to } 3 \mathrm{sec} \text { (vert.) } \\
& \text { velocity sensitivity } 1,85 \text { volts } / \mathrm{cm} / \mathrm{sec} \\
& \text { coil resistance } 380 \mathrm{ohms} \text { with available } 15 \text { ths } \\
& \text { galvanometers period } 0,25 \mathrm{sec} \\
& \text { nominal coil resistance } 50 \mathrm{ohms} \\
& \text { external } 0,6 \text { critical damping resistance } 340 \text { ohms. }
\end{aligned}
$$

It is put into evidence the necessity, for a close surveillance of the volcano activity, of tilmetric, gravimetric and magnetic surveys.

(*) Istituto di Fisica - Istituto di Geologia - Università di Catania. 


\section{Premessa.}

Nello studio dei vulcani sono attualmente impiegate in larga misura le tecniche geofisiche più avanzate, in aggiunta ai tradizionali metodi d'indagine della mineralogia, petrografia, geochimica ecc. Tra di esse è preminente la sismologia, di cui è ormai ben conosciuta l'utilità ai fini dello studio delle relazioni tra attività vulcanica interna ed esterna, dell'individuazione del basamento dell'edificio vulcanico, della ricostruzione dell'edificio stesso, della determinazione della profondità di eventuali focolai e dello studio della crosta terrestre al di sotto del vulcano.

Importantissimo è il fatto che in altri paesi si è giunti con l'ausilio di metodi geofisici ad avvertire con un certo anticipo i prodromi di violente eruzioni, e questo risultato va perseguito anche nel caso nostro.

L'Etna in teoria non è tra i vulcani più pericolosi, poiché la sua attività effusiva si traduce in lento trabocco di colate laviche senza accompagnamento di fenomeni rapidi e violenti quali nubi ardenti, terremoti catastrofici, od altro. Sono però numerose durante la sua storia le colate eccentriche e laterali, di cui sono testimoni i numerosi coni secondari che punteggiano le sue pendici. Basti ricordare fra le altre l'eruzione del 1669 , originatasi in prossimità del centro abitato di Nicolosi e che provocò la parziale distruzione di Catania. In casi come questo un preavviso di pochi giorni o di poche ore puó bastare ad evitare molti danni a persone e cose.

Con questi obbiettivi pratici, certamente non immediati dal momento che l'approfondita conoscenza di un vulcano richiede molti anni di pazienti studi, e con gli obbiettivi scientifici sopra ricordati, si è studiata la realizzazione di una rete di stazioni sismografiche sull'Etna. Ad esse, mezzi finanziari permettendo, dovranno aggiungersi quanto prima stazioni tiltmetriche, gravimetriche e magnetometriche.

Nel mese di Ottobre 1966 è stata completata dagli scriventi l'installazione della prima stazione sismica tra quelle previste nei programmi dell'Istituto di Vulcanologia di Catania diretto dal Prof. Leo Ogniben. La rete completa dovrebbe essere costituita da almeno cinque stazioni disposte in modo tale da formare un allineamento Cataniacratere centrale ed un triangolo approssimativamente equilatero centrato sul cratere stesso (Fig. 1). 


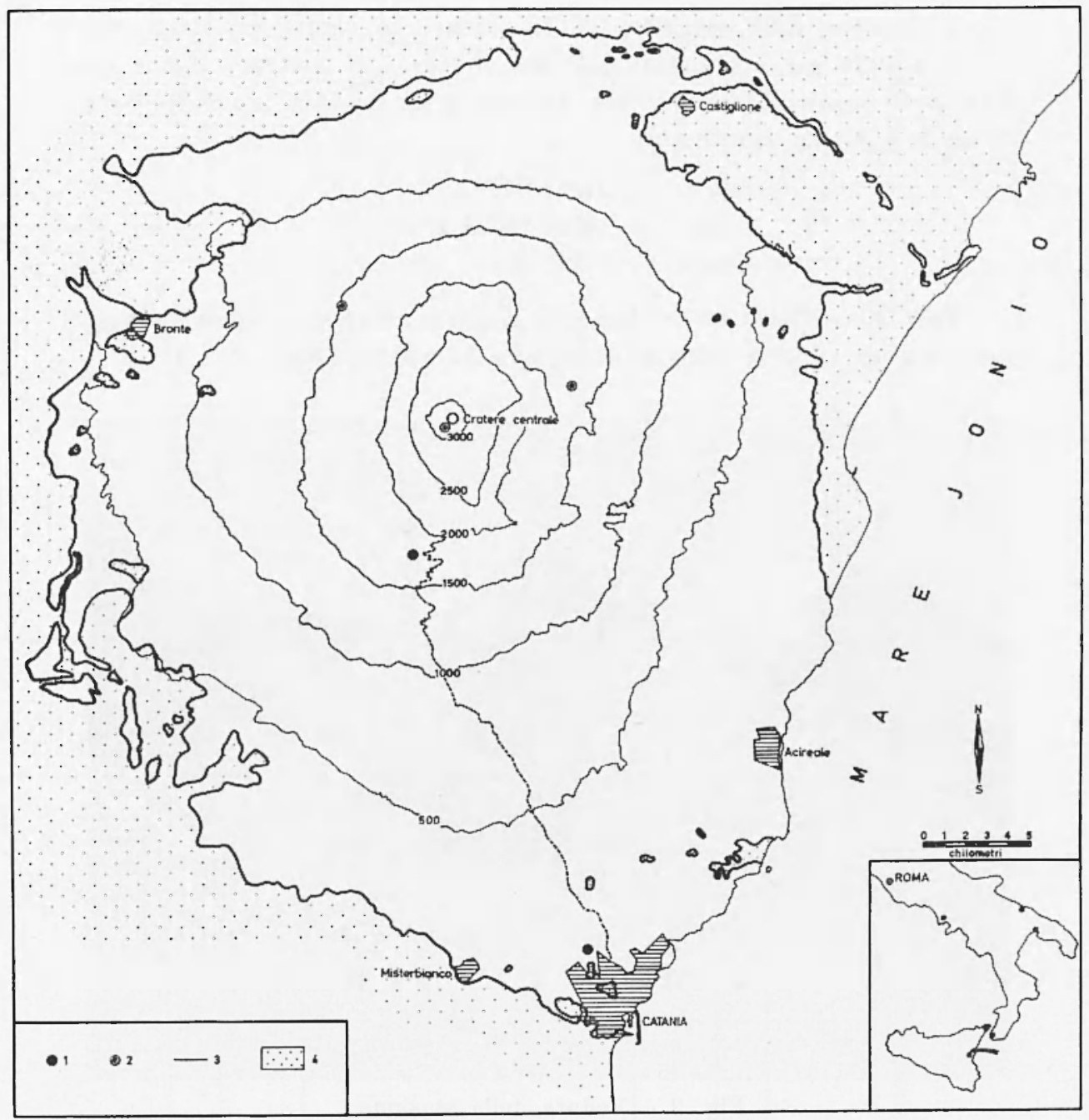

Fig. 1 - Ubicazione delle stazioni sismiche sull'Etna.

- 1 Stazione esistente

- 2 Stazioni in programma
- 3 Limite dell'eruttivo

- 4 Sedimentario 


\section{I. - La Stazione sismica.}

Ubicazione della stazione. - L'edificio che ospita gli strumenti si trova sulle pendici meridionali del vulcano, in contrada Serra La Nave nei pressi dell'Osservatorio Astrofisico di Catania (Sede stellare) ed ha le seguenti coordinate:

$$
\begin{aligned}
& \text { lat. }=37^{\circ} 41^{\prime} 30^{\prime \prime} \mathrm{N} \\
& \text { long. }=14^{\circ} 45^{\prime} 22^{\prime \prime}, 9 \mathrm{E} \\
& \text { quota }=1725 \mathrm{~m}
\end{aligned}
$$

Esso è costituito da tre locali in muratura impermeabilizzata poggrianti su un ripiano intagliato in lava compatta (Figg. 2 e 3). Nel-

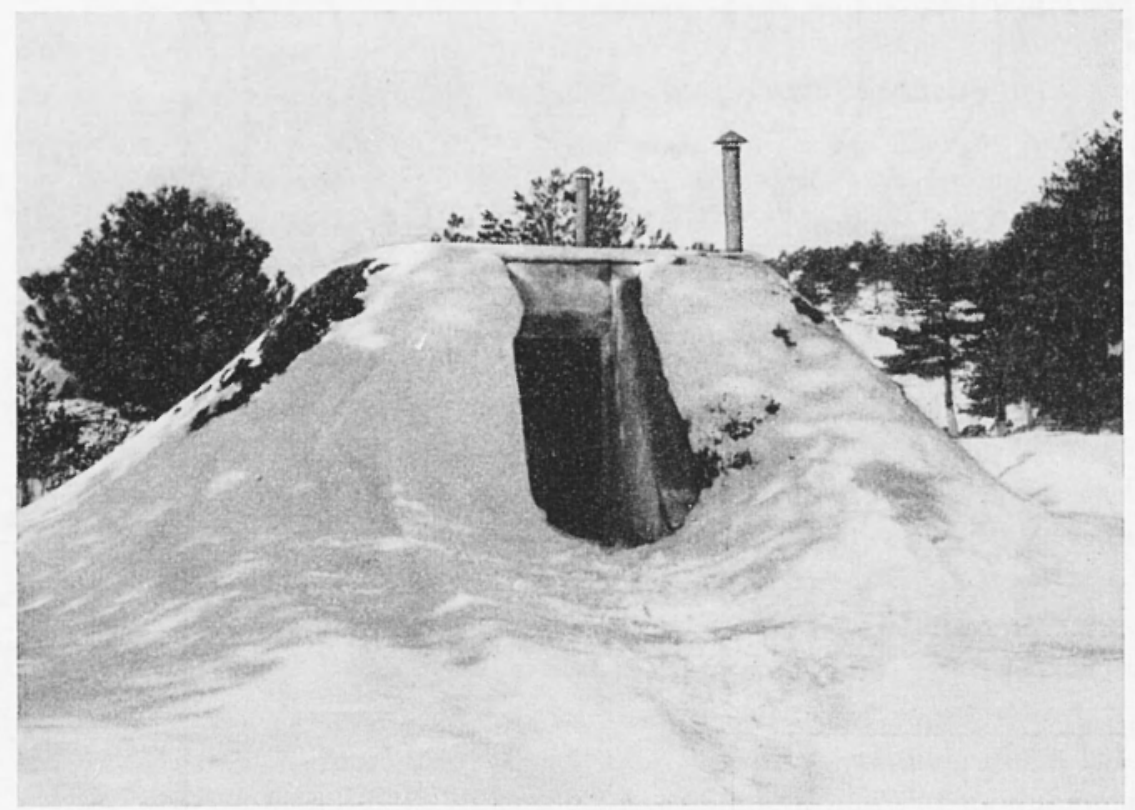

Fig. 2 - Veduta della stazione

l'ambiente principale si trova il basamento in cemento ancorato alla roccia ed isolato dal massetto mediante un'intercapedine d'aria (Fig. 4). Sul basamento sono disposti, i pendoli, i galvanometri ed il registratore (oltre a strumenti meteorologici). Nei due locali minori sono stati disposti un orologio a quarzo, un armadietto per la carta fotosensibile e per il materiale di ricambio, le batterie ed il dispositivo di ricarica 


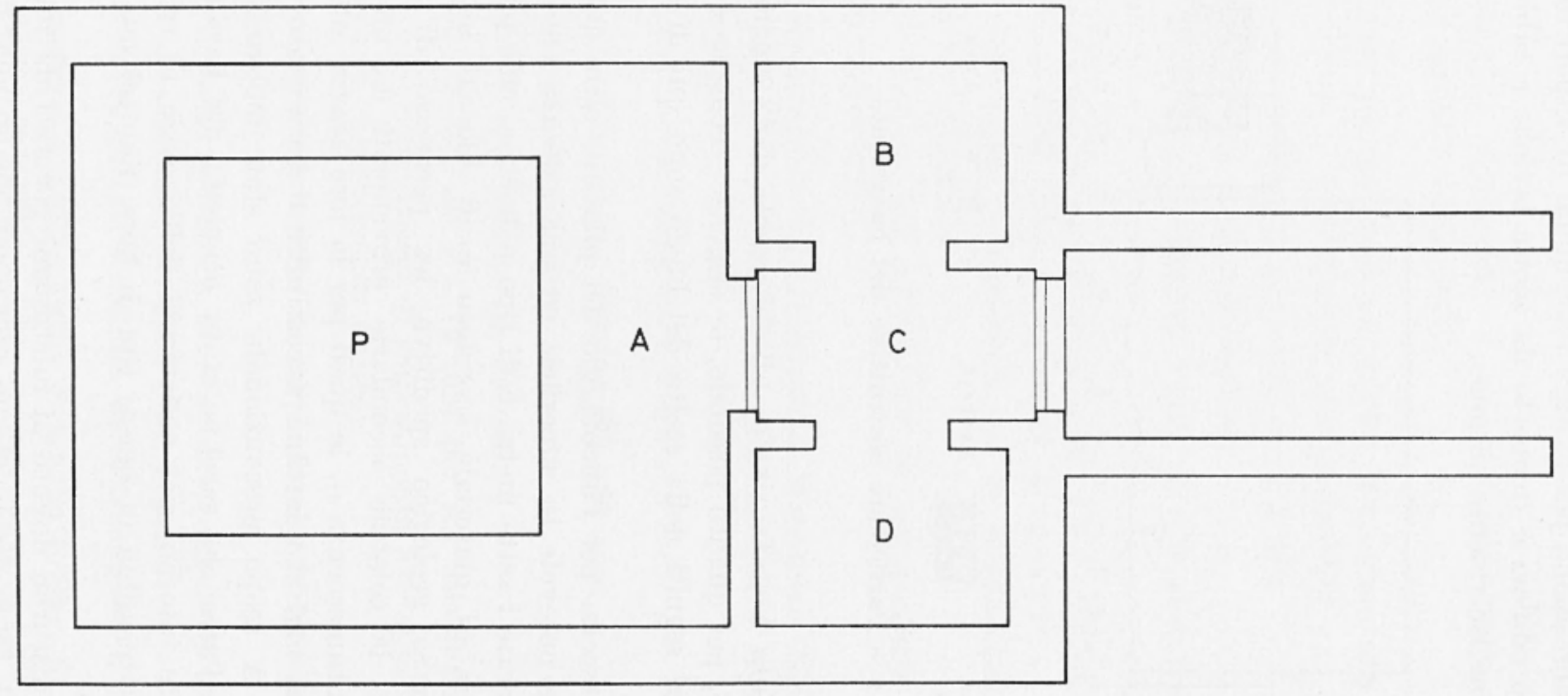

Fig. 3 - Pianta della stazione (Scala $1: 50$ ).

A - Locale principale; B e D - I ocali per strumenti e materiale accessori; C - Ingresso; P - Basamento. 
delle stesse. In particolare l'ambiente ospitante le batterie è dotato di un sistema di aereazione per evitare l'accumulo di vapori di acido solforico. L'intero edificio è ricoperto da scorie laviche e sabbia per assicurarne il buon isolamento termico.

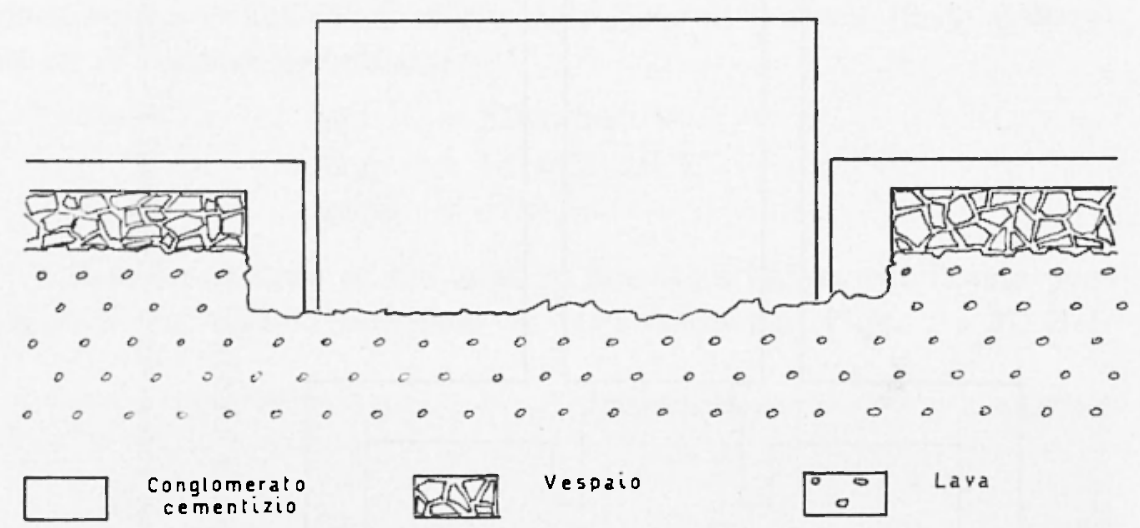

Fig. 4 - Particolare costruttivo del basamento

Particolare cura è stata rivolta all'ubicazione della stazione dovendosi conciliare, per quanto possibile, le esigenze tecniche e quelle logistiche. I criteri seguiti nella scelta del luogo sono qui di seguito esposti.

La colata prescelta per l'ancoraggio del pilastro, oltre a presentare un'estensione notevole in superficie ed una potenza che giunge certamente a superare i sette metri, è di tipo pahoehoe, cioè piuttosto compatta e povera di materiale scoriaceo e di blocchi in superficie $\left({ }^{1}\right)$. Tn rilievo geologico speditivo ha permesso di individuare, ed evitare le colatine secondarie provenienti da un vicino "tumulo" (od “intumescenza "), le quali per la loro scarsa estensione e limitata potenza mal si adattano a costituire il basamento di una stazione sismica. A scopo precauzionale sono stati inoltre eseguiti nove sondaggi nell'area che sarebbe stata occupata dal basamento e nel suo immediato intorno, per assicurarsi dell'assenza di cavità di grandi dimensioni, possibili in questi tipi di lave, fino ad una ragionevole profonditì.

L'entitì del distarbo dovuto ai microsismi generati da moto ondoso (la costa del Jonio dista nel suo punto più vicino non meno di $18 \mathrm{~km}$ ), dal vento e da attività umane, è contenuta entro limiti ac- 
cettabili. La località è collegata a Catania da rotabile, telefono e linea elettrica.

Dotazione strumentale. - La dotazione strumentale comprende per ora i seguenti strumenti:

a) due pendoli elettromagnetici WILLMore MK II (Fig. 5) orizzontali aventi le sottoelencate caratteristiche:

- periodo regolabile da $0,75 \mathrm{sec}$ a $5 \mathrm{sec}$;

— sensibilità 1,85 volts $/ \mathrm{cm} / \mathrm{sec}$;

- bobina con resistenza totale di 380 ohm frazionabile in quindicesimi;

- intervallo di temperatura utile per il funzionamento a periodo di 3 see $\pm 50^{\circ} \mathrm{C}$ della temperatura di taratura;

- variazione di sensibilità con la temperatura inferiore al $5 \%$ (sull'intero intervallo);

- variazione del periodo con la temperatura inferiore al $10 \%$ (sull'intero intervallo).

b) un pendolo elettromagnetico WILLMone MK II (Fig. 5) verticale avente caratteristiche tecniche identiche a quelle sopra enumerate tranne che per:

- periodo variabile da 0,6 sec a 3 sec.

c) tre galvanometri Tinsley SG 431 con:

- periodo di $0,25 \mathrm{sec}$;

- resistenza interna da $50 \mathrm{ohm}$;

— sensibilità da 8 a $12 \mathrm{~mm}$ per $\mu A$ alla distanza di $10 \mathrm{~cm}$;

- resistenza esterna di smorzamento 340 ohm (pari ai $6 / 10$ della resistenza di smorzamento critico).

d) registratore ottico a carta fotosensibile con le seguenti caratteristiche: (1)

- tamburo cilindrico con circonferenza di $90 \mathrm{~cm}$;

- velocitì periferica di 6 o $12 \mathrm{~cm} / \mathrm{min}$;

- trascinamento mediante motore sincrono Philips da 6 watts.

e) orologio a quarzo Patek ECZK 24 R (Fig. 6) di caratteristicle:

- errore inferiore a 0,01 see per giorno a $20^{\circ} \mathrm{C}$;

- errore inferiore a $0,1 \mathrm{sec}$ per giorno tra $15^{\circ}$ e $25^{\circ} \mathrm{C}$. 


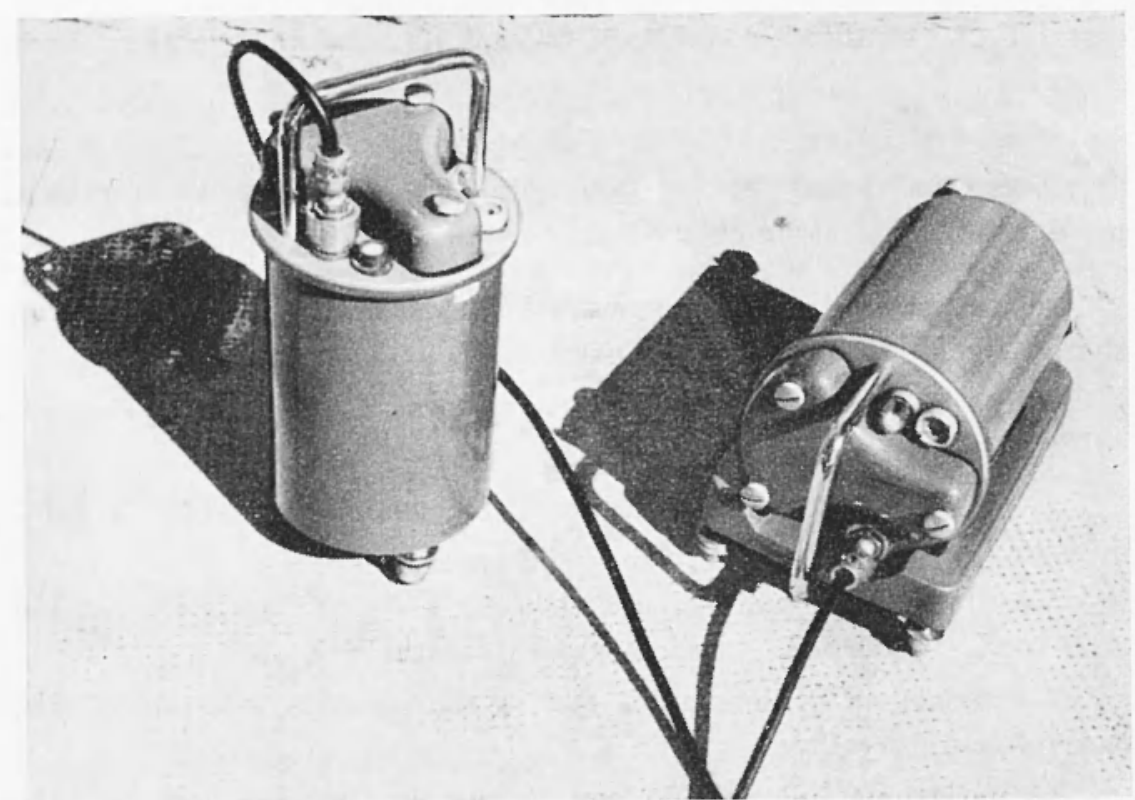

Fig. 5 - I pendoli elettromagnetici Willmore MK II.

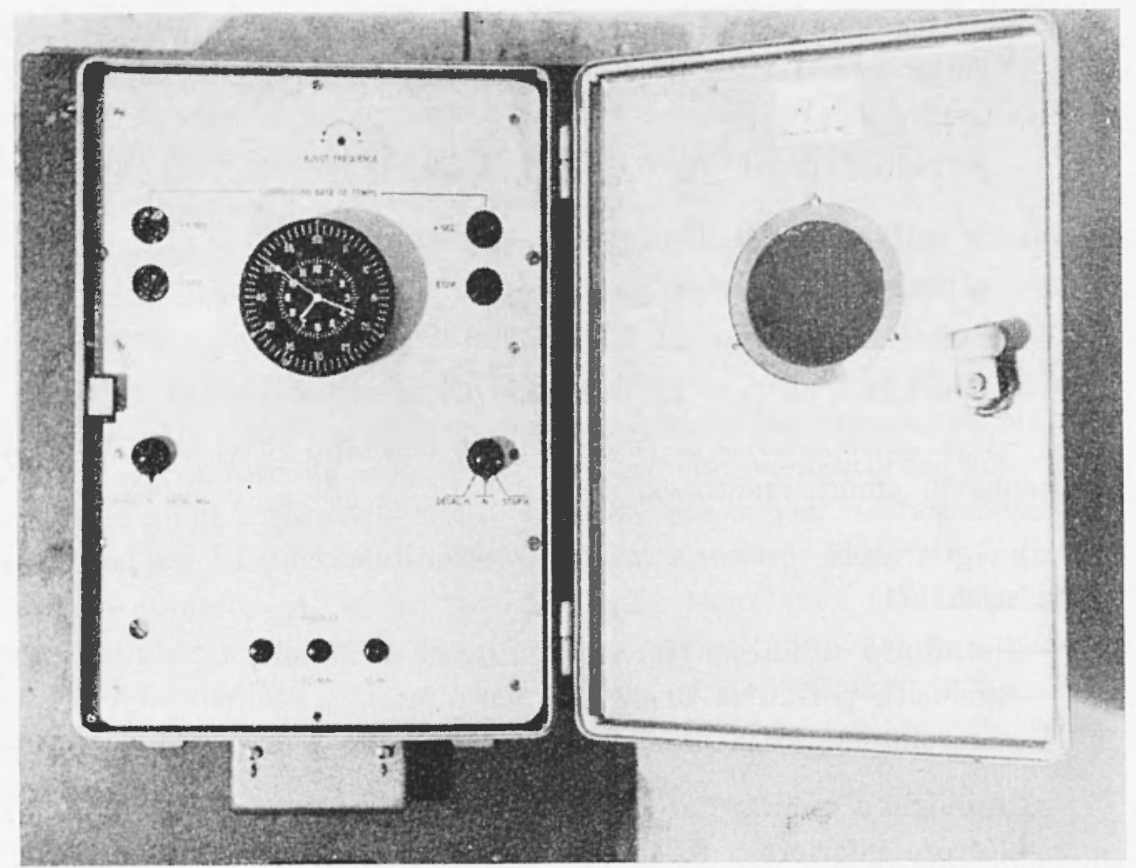

Fig. 6 - Orologio a quarzo. 
f) apparecchio radio ricevente da $75 \mathrm{KHz}$ per la ricezione dei segnali orari.

g) oscillografo Philips per la sincronizzazione dei segnali della radio e dell'orologio.

h) sistema di alimentazione a batteria da 24 volts con dispositivo di ricarica automatica dalla rete (Fig. 7).

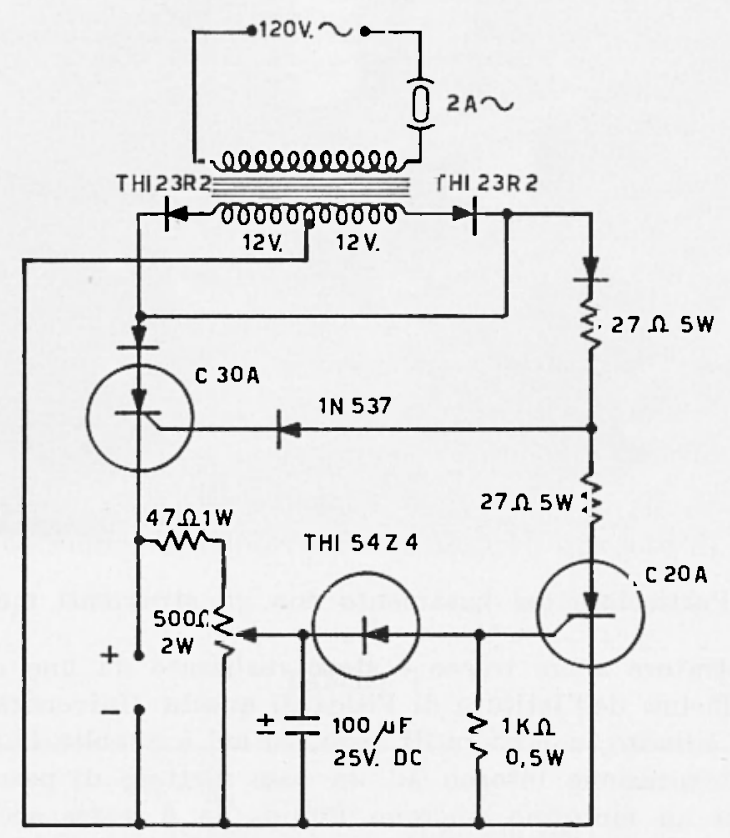

Fig. 7 - Schema del sistema di ricarica delle batterio.

La strumentazione è completata da una terna di strumenti meteorologici Salmoiraghi per la rilevazione dei valori di temperatura, umiditì e pressione all'interno del locale (Fig. 8).

E allo studio la realizzazione di un sistema deumidificatore per ovviare agli inconvenienti dovuti all'alta umidità ambiente soprattutto nella stagione invernale.

Cenni sulle caratteristiche del sismografo Willmore MK II. Lo studio dell'equazione del moto di un pendolo, quando quisto è 
connesso elettricamente ad un galvanometro, conduce nel caso dei Willınore MK II $\left(^{2}\right)$ ad una relazione fra i parametri caratteristici

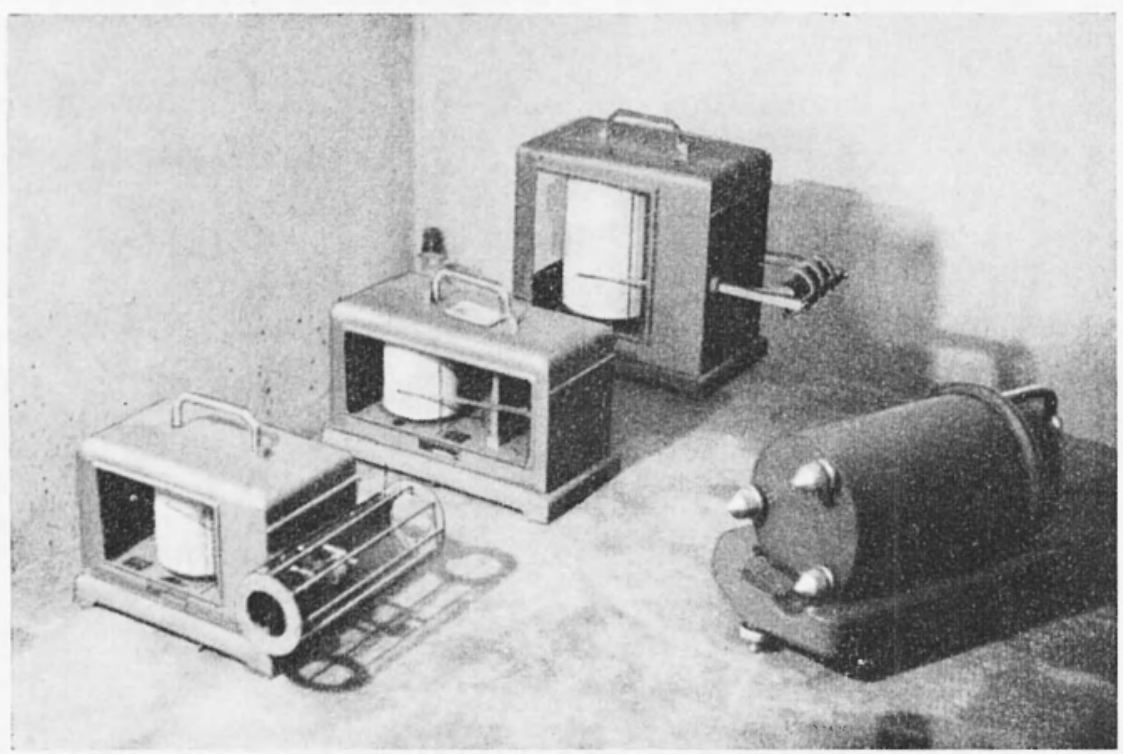

Fig. 8 - Particolare del basamento con gli strumenti meteorologici.

Il registratore a tre tracce è stato realizzato da uno degli scriventi (A.B.) nell'officina dell'Istituto di Fisica di questa Universitì. Esso è composto da un cilindro in lamiera di ferro, su cui è avvolta la carta fotosen. sibile, la cui rotazione intorno ad un asse filettato di passo $2,5 \mathrm{~mm}$ è assicurata da un motorino sincrono Philips da 6 watts accoppiato al cilindro mediante una scatola di riduzione da $3750: 1$ (o da $1875: 1$ ), che porta i 250 giri/min. dell'asse del motorino ai 4 giri/h (o 8 giri $/ \mathrm{h}$ ) del cilindro registratore.

Una coppia di chiavi operanti sulla filettatura dell'asse di questo ultimo provoca la traslazione assiale del cilindro che consente la migliore utilizzazione della superficie impressionabile.

Una custodia metallica parzialmente apribile a compasso consente le operazioni di sostituzione della carta e la manovra del dispositivo per la messa in folle del cilindro durante le operazioni stesse. Sulla parte frontale della custodia sono praticate tre finestre rettangolari in cui sono alloggiate mediante intelaiature tre lenti semicilindriche a posizione regolabile per la messa a fuoco sulla carta registrante dei fascetti luminosi provenienti dagli specchietti dei galvanometri.

Il basamento rigido dell'apparato registratore è realizzato mediante un telaio in profilato di ferro a sezione rettangolare. Quattro robuste viti calanti permettono la livellazione dello strumento. 
del sistema stesso come massa $M$, periodo delle oscillazioni libere $T$, smorzamento $D$, fattore $K$ e resistenza totale del circuito $R$ :

$$
D=\frac{T}{4 \pi M} \cdot \frac{K^{2}}{R} \cdot 10^{7}+A T
$$

con:

$$
R=R_{a}+R_{b}+R_{g}+R_{s}
$$

dove $A$ è un coefficiente che esprime lo smorzamento dovuto a parti esterne al circuito. Per questi sismografi il valore di $A$ è circa $1 \% 1 \%$ del valore di $K^{2} / R_{b}\left(R_{b}=\right.$ resistenza della bobina $)$.

Una volta scelto il galvanometro da accoppiare al sismometro e quindi il suo grarlo di smolzamento, la resistenza totale del circuito $(R)$ è automaticamente definita. Successivamente, scelti i valori più idonei per il periodo $T$ e per il fattore di smorzamento $D$, resta individuato il valore di $K$. Con i sismometri Willmore MK II, questa non è una condizione restrittiva poiché la possibilità di usare frazioni della bobina consente di disporre di un numero discreto di valori di $K$ (Tabella I).

Tabella I

\begin{tabular}{|c|c|c|c|}
\hline$\frac{R D}{T}$ & $\begin{array}{c}R_{b} \\
(0 \mathrm{hm})\end{array}$ & $\begin{array}{c}K \\
\text { volts/cm/sec }\end{array}$ & $\begin{array}{c}\text { Frazioni di bobina } \\
\text { espresse } \\
\text { in quindicesimi }\end{array}$ \\
\cline { 2 - 4 } 22 & 80 & 0,37 & 3 \\
40 & 100 & 0,49 & 4 \\
60 & 130 & 0,61 & 5 \\
90 & 150 & 0,74 & 6 \\
120 & 180 & 0,86 & 7 \\
160 & 200 & 0.98 & 8 \\
200 & 230 & 1,11 & 9 \\
250 & 250 & 1,23 & 10 \\
300 & 280 & 1,35 & 11 \\
360 & 300 & 1,47 & 12 \\
425 & 330 & 1,60 & 13 \\
560 & 380 & 1,85 & 15 \\
\hline
\end{tabular}


Tali valori sono stati calcolati tramite la [1] in cui è stato trascurato il termine $A T$ del secondo membro.

Nel caso in cui si debbano utilizzare valori di $T$ per cui risultino valori di $R D / T$ intermedi tra quelli riportati in Tab. I si può ricorrere ad una resistenza di shunt $\left(R_{8}\right)$ posta in serie con la bobina del sismometro come schematizzato in Fig. 9.

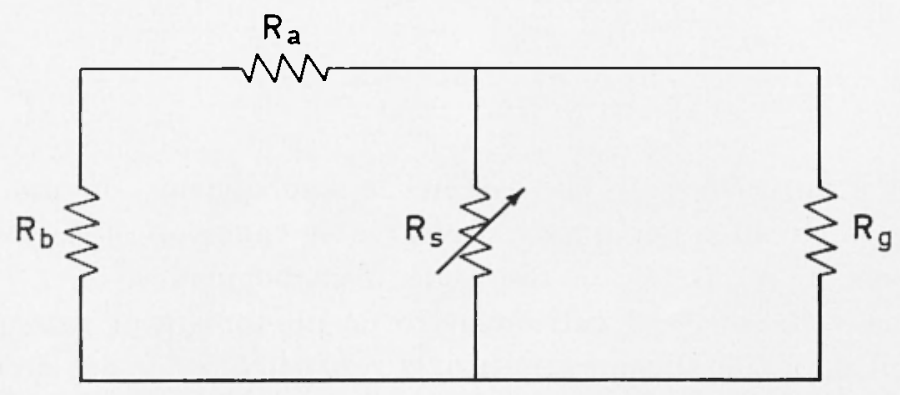

Fig. 9 - Circuito sismo-galvanometrico.

dove $R_{b}$ ò la resistenza della frazione di bobina sismometrica utilizzata; $R_{g}$ è la resistenza del galvanometro:

$R_{a}$ è la resistenza necessaria per portare il circuito alle condizioni di smorzamento voluto;

$R_{s}$ è la resistenza di shunt.

In Figg. 10 e 11 sono rappresentate rispettivamente le curve di risposta e di amplificazione dinamica per $\mathrm{i}$ sismometri in questione.

\section{II. - APPLICAZIONI vUlCANOLOGICHE.}

Microsismi e terremoti vulcanici. - Il massimo sviluppo attuale della sismologia vulcanica si ha in Giappone, ove all'alta concentrazione di vulcani attivi si aggiunge la loro grande pericolosità. Come conseguenza di questo stato di cose sono sorti da tempo, a volte proprio a seguito di avvenimenti particolarmente catastrofici, numerosi istituti che hanno il compito di studiare, sorvegliare e possibilmente predire con ragionevole anticipo le eruzioni e le manifestazioni ad esse associate.

Nel campo della sismologia studiosi $\left({ }^{3}\right)$ si sono intessati da tempo alla ricerca di correlazioni tra attività effusiva, struttura dei vulcani, 


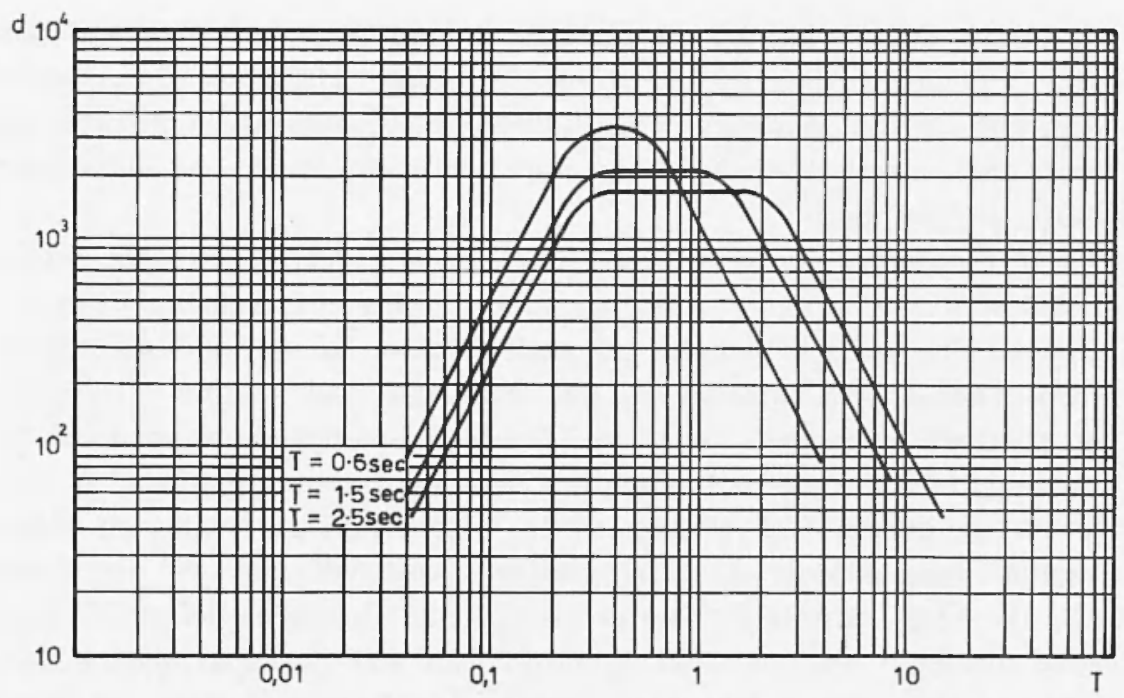

Fig. 10 - Curve di risposta del pendolo Willmore MK II con galvanometri da 0,25 sec

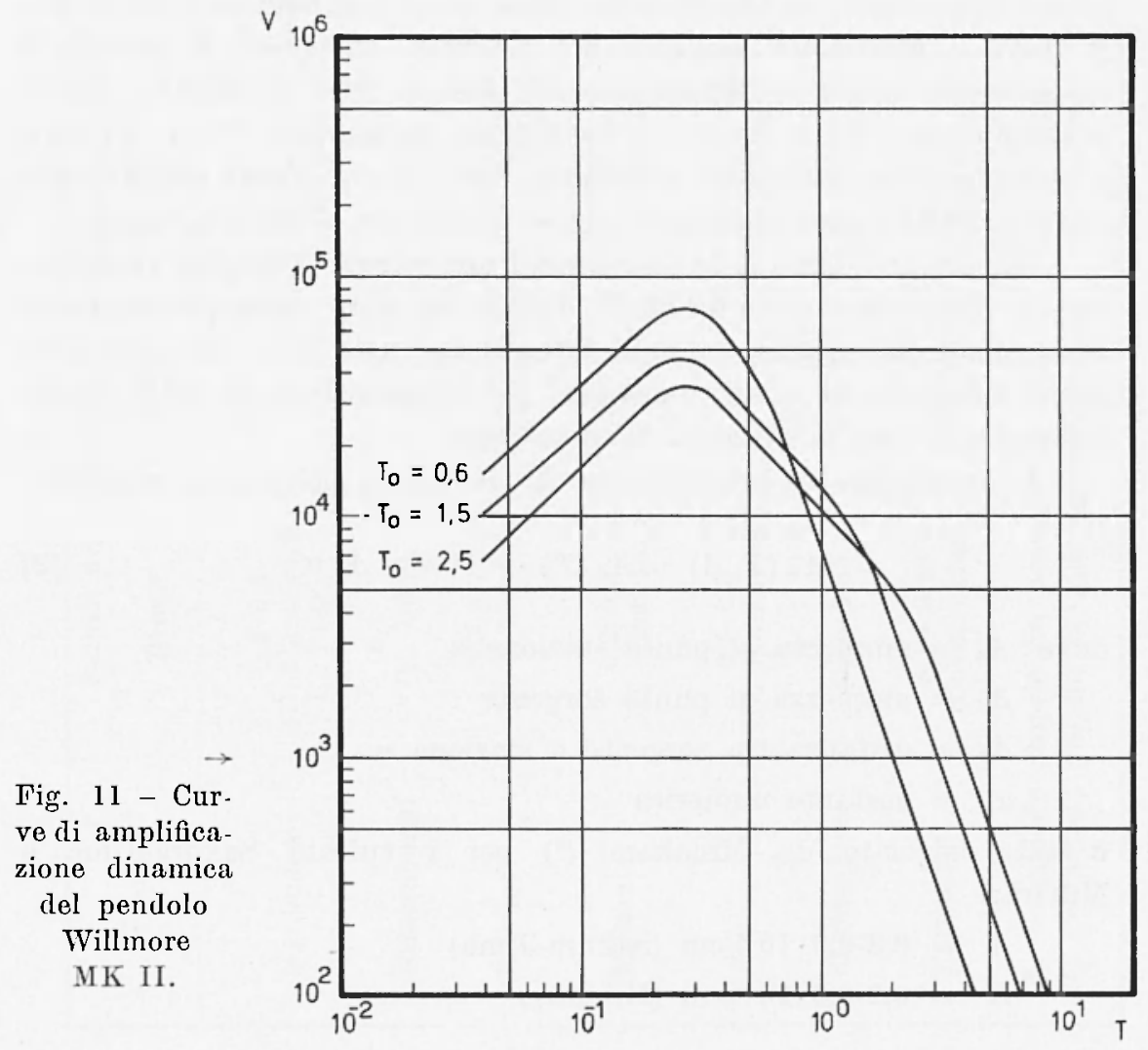


proprietà chimico-fisiche dei magmi e manifestazioni sismiche nelle aree vulcaniche. Per ciò che riguarda queste ultime sembra esservi oggi una diffusa tendenza a considerare separatamente agitazione microsismica e terremoti, considerandoli fenomeni distinti ed entro certi limiti indipendenti.

I parametri che meglio si prestano a caratterizzare un microsisma sono periodo ed ampiezza e subordinatamente velocità di propagazione. Secondo Minakami $\left({ }^{4}\right)$ nello studio del periodo dei microsismi non si puó prescindere dal chimismo dei magmi (Tab. II); in particolare periodi maggiori sarebbero sempre corrispondenti ad un magmatismo basico e quindi a lave relativamente fluide. $A$ conferma di questa tesi stanno ovvie considerazioni fisiche ed osservarioni sperimentali di altri studiosi, tuttavia non va sottaciuto che da altri Autori $\left(^{5}\right)$ le variazioni del periodo dei microsismi sono messe in relazione con la profondita della zona di origine delle lave, e quindi con la fase di attività del vulcano. E stato osservato infatti che durante le fasi di quiescenza, quando il magma ovviamente si trova a profondità maggiori nel condotto vulcanico, il periodo è maggiore di quello corrispondente alle fasi di attività effusiva. Molto probabilmente i due fenomeni coesistono, prevalendo l'uno o l'altro a seconda delle particolari condizioni locali e dei criteri seguiti nelle misure. Tutti questi studi riguardano microsismi a breve periodo.

Per ciò che riguarda le ampiezze, i microsismi di origine vulcanica non si discostano molto da quelli dovuti ad altre cause (vento, moto ondoso, attività umane, ecc.) che interessano vaste aree continentali $\left(^{\circ}\right)$ ma a differenza di questi mostrano la caratteristica di una rapida attenuazione con la distanza dalla sorgente.

Il coefficiente di attenuazione $K$ che figura nella nota relazione:

$$
A_{n}(T, \Delta)=A_{o}(T) \cdot \rho_{0}^{-K(T) \Delta} \cdot A_{n}^{-\alpha}
$$

dove $A_{n}=$ ampiezza al punto stazione $n$

$A_{0}=$ ampiezza al punto sorgente

$A_{n}$-- distanza tra sorgente e stazione $n$

$\alpha=$ costante numerica

è stato calcolato da Minakami ${ }^{4}$ ) per i vuleani Sakura-Jima e Mihara:

$$
\begin{aligned}
& K=0,8-0,9 \cdot 10^{-5} / \mathrm{cm} \text { (Sakura-Jima) } \\
& K=0,2-0,3 \cdot 10^{-5} / \mathrm{cm} \text { (Mihara) }
\end{aligned}
$$


Tabella II

\begin{tabular}{|c|c|c|c|c|c|c|}
\hline \multirow[b]{2}{*}{ Vulcani } & \multicolumn{2}{|c|}{ Periodi dei microsismi } & \multirow{2}{*}{$\begin{array}{c}\text { Contenuto medio } \\
\text { di silicati } \\
\% \\
\end{array}$} & \multirow{2}{*}{$\begin{array}{c}\text { Temperature } \\
\text { delle lave } \\
\left({ }^{\circ} \mathrm{C}\right)\end{array}$} & \multirow{2}{*}{$\begin{array}{c}\text { Viscosità } \\
\text { delle lave } \\
\text { (c.g.s.) }\end{array}$} & \multirow{2}{*}{$\begin{array}{c}\text { Periodo } \\
\text { dei sismografi } \\
\text { (sec) }\end{array}$} \\
\hline & Brevi & $\underset{\text { (sec) }}{\text { Lunghi }}$ & & & & \\
\hline Nyragongo & $\begin{array}{l}0,3-0,5 \\
\text { (D. Shimozuru, } \\
\text { E. Berg) }\end{array}$ & - & $\begin{array}{l}\stackrel{40}{\text { (T. G. Sahama, }} \\
\text { A. Meyer) }\end{array}$ & $\begin{array}{c}1095 \\
\text { (J. Verhoogen) }\end{array}$ & - & $\begin{array}{l}T_{o}=1,0 \\
T_{g}=0,3\end{array}$ \\
\hline Kitzembanyi & (E. Berg) & 一 & 40 & - & 一 & $\begin{array}{l}T_{o}=1,0 \\
T_{g}=0,25\end{array}$ \\
\hline Kilauea & $\begin{array}{l}\stackrel{0,5-0,7}{\text { (J. Eaton) }}\end{array}$ & $2,5-3,5$ & $\begin{array}{l}\text { (J. P. Eaton, } \\
\text { D. H. Richter) }\end{array}$ & $\begin{array}{c}1120-1190 \\
\text { (J. P. Eaton) } \\
\text { D. H. Richter) }\end{array}$ & 一 & $\begin{array}{l}T_{o}=0,5 \\
T_{g}=0,5\end{array}$ \\
\hline Mihara & (T. Minakami) & - & $\begin{array}{c}52 \\
\text { (I. Iwasaki) }\end{array}$ & $\begin{array}{c}950-1100 \\
\text { (T. Minakami) }\end{array}$ & $\begin{array}{l}10^{3} \\
\text { (R. Takahasi, } \\
\text { D. Shimozuru) }\end{array}$ & $\begin{array}{l}T_{o}=1,0 \\
T_{\theta}=0,3\end{array}$ \\
\hline Aso & (D. Shimozuru) & - & $\begin{array}{c}53 \\
\text { (S. Taneda) }\end{array}$ & 一 & - & $\begin{array}{l}T_{o}=1,0 \\
T_{\theta}=0,3\end{array}$ \\
\hline Sakura-Jima & $0,25-0,35$ & - & $\begin{array}{c}60 \\
\text { (S. Taneda) }\end{array}$ & (T. Nagata) & $\begin{array}{c}10^{8}-10^{9} \\
\text { (T. Nagata) }\end{array}$ & $\begin{array}{l}T_{o}=1,0 \\
T_{\theta}^{\prime}=0,3\end{array}$ \\
\hline Paricutin & $\begin{array}{c}0,10-0,20 \\
\text { (Covarrubias) }\end{array}$ & $0,35-0,60$ & $\begin{array}{c}55-59 \\
\text { (R. E. Wilcox) }\end{array}$ & $\begin{array}{c}1050-1070 \\
\text { (Krauskopf) }\end{array}$ & $\begin{array}{c}10^{5}-10^{6} \\
\text { (Krauskopf) }\end{array}$ & 一 \\
\hline
\end{tabular}


avendo attribuito ad $\alpha$ il valore $1 / 2$ che costituisce il limite inferiore dell'intervallo, $1 / 2 \leqslant a \leqslant 1$ entro cui varia $a$ in funzione del periodo: 1 valore $1 / 2$ è relativo alle onde superficiali di lungo periodo ed il valore 1 (circa) è proprio delle onde longitudinali di breve periodo $\left({ }^{7}\right)$.

Queste considerazioni sono naturalmente di carattere generale e non possono applicarsi indipendentemente dalle caratteristiche strutturali di ciascun vulcano (accumuli di materiale piroclastico incoerente, fessurazioni, ecc.). Tali strutture influenzano in misura notevole la velocità di propagazione delle onde sismiche come appare evidente dall'analisi dei dati noti. Per il vulcano Aso si hanno infatti valori che a seconda dell'ubicazione della stazione registratrice variano da 0,6 a $4 \mathrm{~km} / \mathrm{sec}$. Minore dispersione si ha per il Sakura-Jima dove le basse velocità del'ordine di $0,6-0,8 \mathrm{~km} / \mathrm{sec}$ sono da mettere in relazione con l'abbondanza di lave a blocchi e materiale piroclastico sciolto $\left(^{4}\right)$.

Più proficuo sembra essere ai fini della previsione delle eruzioni lo studio dei terremoti, in quanto essendo essa basata su dati statistici, ci si può avvalere di una più cospicua messe di dati potendo estendere l'indagine anche ai periodi precedenti la nascita della moderna sismologia vulcanica. In questo campo il risultato più vistoso è stato ottenuto nel 1958 quando fu prevista, con circa un mese di anticipo, l'eruzione dell'Asama sulla base di una legge statistica elaborata in base a dati laccolti in 25 anni di osservazioni continue ${ }^{8}$ ). In gran numero di casi è stato osservato prima dell'eruzione un intensificarsi dell'attività sismica e dell'emissione di fumi. L'interpretazione statistica dei dati inerenti alle fasi successive all'eruzione si presenta complessa ed allo stato attuale non è possibile stabilire una relazione tra i due tipi di manifestazioni vulcaniche, cioè microsismi e terremoti.

In relazione alla profondità ipocentrale Minakami ed altri $\left(^{8}\right)$ distinguono quattro tipi di manifestazioni sismiche:

a) "volcanic pulsations" costituite da treni di oscillazioni armoniche che hanno origine nella zona del cratere terminale;

b) terremoti di tipo $B$ che hanno origine nelle parti superiori del vulcano; essi sembrano strettamente associati all'attività eruttiva. Al Sakura-Jima è stato osservato come fenomeno generale l'iniziarsi di questo tipo di attività con un anticipo di 10-15 giorni sulla data delle eruzioni;

c) terremoti associati ad eruzioni esplosive; 
d) terremoti di tipo $A$ con ipocentri profondi qualche chilometro e relativamente poco frequenti.

La situazione si presenta differente nel distretto delle Hawaii, dove la diversità di caratteristiche magmatologiche di questi vulcani rispetto a quelli giapponesi si traduce in diversità di attività sismica. Mac Donald ed Eaton $\left(^{\circ}\right)$ hanno descritto con ricchezza di dettagli le eruzioni del Kilauea del 1955, distinguendo due stadi dell'eruzione: un primo stadio in cui il magma risalente colma il condotto vulcanico e gli eventuali serbatoi crustali, generando pressioni sulle pareti che lo racchiudono sino a provocare prima deformazioni e poi fratture dalle quali fuoriesce il magma, ed un secondo stadio in cui avviene il parziale collasso delle rocce incassanti e della caldera in seguito allo svuotamento provocato dall'eruzione.

Mac Donald ed Eaton sono giunti a queste conclusioni utilizzando i dati delle registrazioni sismiche e clinografiche. Queste ultime si sono rivelate particolarmente efficaci per seguire le fasi precedenti l'eruzione, quando le pressioni generali della risalita del magma sono immagazzinate elasticamente dalle rocce incassanti, e gli unici terremoti avvertibili sono quelli generati dai movimenti del magma risalente. Questo genere di scosse ha inizio anche qualche anno prima dell'eruzione e vi è un progressivo intensificarsi del fenomeno nei giorni ad essa immediatamente precedenti.

In concomitanza con l'eruzione sono stati registrati tremori armonici di due tipi distinti: con periodo di 0,8-1 sec durante la fase effusiva e con periodi di $0,3-0,5$ sec durante le fasi intermedie in eui viene a mancare l'attività effusiva. Questi ultimi, a differenza dei primi, hanno ipocentri profondi qualche chilometro.

Una situazione per alcuni aspetti analoga a quella dei vulcani giapponesi si ha al Nyiragongo (Congo) $\left(^{7}\right)$. Per quanto riguarda il periodo dei microsismi è rilevabile la dipendenza dal chimismo del magma e l'effetto apparentemente trascurabile della lunghezza del tragitto delle onde.

Inoltre, la distribuzione delle frequenze sembra essere correlabile con le fasi di attività effusiva; in particolare si riscontra una diminuzione delle frequenze in corrispondenza alle fasi di quiescenza.

Secondo Blot e Tazieff $\left({ }^{10}\right)$, invece, la dipendenza del periodo dell'agitazione microsismica dalla distanza sembra netta al vulcano Tamna (Nuove Ebridi) (Fig. 12). 
Qui è stato inoltre possibile distinguere fra i terremoti associati con attivitì esplosiva, con periodi da 0,5 a 0,8 sec, e terremoti profondi da 2 a $4 \mathrm{~km}$ con periodi da 0,2 a 0,4 sec. Questi due tipi di terremoti possono essere riconosciuti, nella classificazione di Minakami sopra riportata, per quelli menzionati rispettivamente alle lettere c) e d).

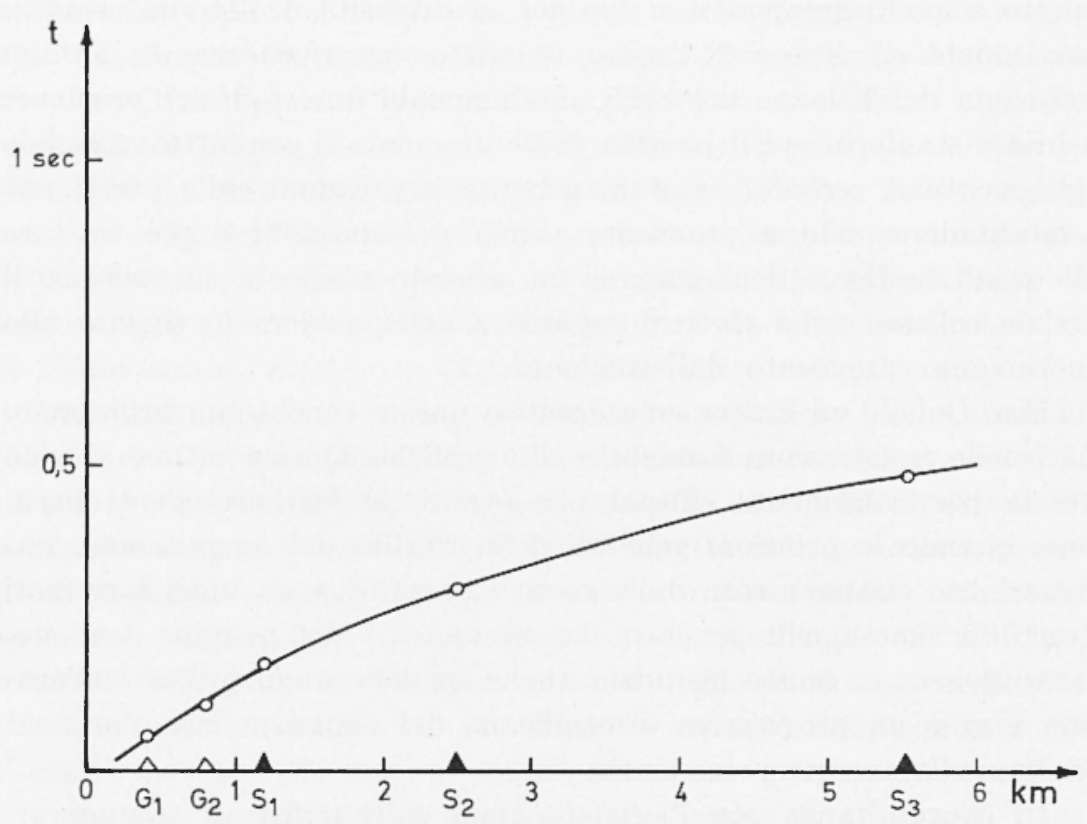

Fig. 12 - Andamento del periodo predominante dei microsismi in funzione della distanza dall'epicentro (da Blot e Tazieff, 1961).

In Italia gli studi vulcanologici condotti con l'ausilio dei metodi geofisici riguardano, tranne casi sporadici, solamente il Vesuvio che è attualmente in fase di quiescenza. Sugli altri vulcani attivi (Stromboli, Vulcano, Etna) sono stati condotti solo saltuariamente e per brevi periodi studi geofisici. Nel complesso la gran mole esistente di studi vulcanologici è stata eseguita con metodi prevalentemente mineralogici, petrografici e geochimici.

I'attività sismica del Vesuvio è caratterizzata da tre tipi fondamentali di manifestazioni: microsismi armonici, microsismi spasmodici ed agritazione sismica (Imbò, 1952). I microsismi armonici sarebbero 
connessi con il libero degassamento delle masse magmatiche nel condotto eruttivo; degassamento che in prossimità della superficie libera, per effetto delle mutate condizioni fisiche ambientali, assume il carattere di ritmiche pulsazioni contro le pareti del condotto. Tale attività è caratterizzata da un andamento continuo e con ampiezza in genere dipendente dall'altezza della colonna magmatica, dalle caratteristiche chimico-fisiche del magma e dalla geometria della parte terminale dell'edificio vulcanico. I microsismi spasmodici presentano invece carattere di intermittenza e sono legati all'attività esplosiva; essi hanno ipocentro a profondità variabile ma in genere superficiale, e per alcuni caratteri possono essere assimilati a sismi veri e propri a fuoco superficiale.

Infine l'agitazione sismica ̀̀ da Imbò descritta come saltuaria, con addensamenti particolari che devono essere posti in relazione con fenomeni di frattura e di crollo interessanti l'edificio vulcanico. I vari tipi di sismi sono inoltre distinti in profondi e superficiali in relazione all'ubicazione dell'ipocentro.

Da quanto riportato appare evidente come, almeno dal punto di vista qualitativo, l'attività sismica del Vesuvio sia assimilabile a quella di alcuni vulcani giapponesi più che a quella dei vulcani hawaiiani.

Lineamenti strutturali dell'Etna. - L'Etna è il vulcano attivo più meridionale d'Europa; posto al centro del Mediterraneo, ha come coordinate geografiche del suo cratere centrale: long. $14^{\circ} 59^{\prime} 38^{\prime \prime} \mathrm{E}$; lat. $37^{\circ} 45^{\prime} 00^{\prime \prime} \mathrm{N}$. L'edificio del cratere centrale Mongibello supera $\mathrm{i}$ $3.200 \mathrm{~m}$ di quota, con variazioni dell'ordine del centinaio di metri per effetto di accumuli e crolli connessi all'attività effusiva. Attualmente è in fase eruttiva anche un secondo cratere posto a Nord-Est di quello centrale, i cui prodotti vanno a raccogliersi entro la valle del Leone a circa $12 \mathrm{~km}$ verso Sud-Est, per poi traboccare verso la valle del Bove, grande caldera relitto di un precedente apparato eruttivo detto il Trifoglietto ( $\left.{ }^{1}\right)$.

In pianta la forma del complesso edificio vulcanico è grosso modo ellittica, con asse maggiore di circa $4 \tilde{5} \mathrm{~km}$ orientato in senso meridiano ed asse minore di circa $37 \mathrm{~km}$.

L'età dell'Etna è relativamente recente, potendosi ascrivere l'inizio della sua formazione ad epoca posteriore alla emersione dei terreni del Siciliano.

Lo spessore effettivo massimo dell'accumulo lavico non corrisponde alla quota del cratere centrale, essendo il vulcano impostato su 
uno zoccolo sedimentario che affiora ai suoi margini sino ad oltre $1000 \mathrm{~m}$ a Nord e Nord-Est. La topografia del basamento sedimentario sottostante l'Etna è stata in questi ultimi tempi indagata soprattutto per ragioni idrogeologiche, con risultati pubblicati a piccola scala (11), secondo i quali non è giustificato supporre la presenza di un horst etneo per spiegare le quote ragrgiunte dal basamento sedimentario, poiché queste ben si inquadrano nel generale sollevamento dell'Isola, e alla loro conservazione concorse la funzione protettrice esercitata dai mantelli lavici nei confronti dell'erosione.

La struttura dell'edificio vulcanico è molto complessa, perchè i prodotti effusivi sono irregolarmente disposti in manti di varia natura ripetutamente intercalantisi. Si hanno anzitutto lave di due tipi principali: "pahoehoe» e " $a a$ ", la cui differenza sostanziale risiede nella gran abbondanza di materiale scoriaceo che ricopre le " $a a$ ", mentre è quasi totalmente assente nelle "pahoehoe" che presentano una superficie superiore relativamente liscia. Inoltre, gran quantità di materiale piroclastico, più o meno cementato, è sovrapposto ed interposto alle colate, come è messo in luce anche dai sondaggi per ricerche d'acqua. Internamente ciascuna colata rresenta generalmente numerose cavità la cui formazione è connessa alle modalità di messa in posto e raffreddamento. La magrgior parte di esse sono di dimensioni modeste, da frazioni di centimetro a qualche centimetro, ma non mancano numerose cavità con dimensioni misurabili a metri.

Impiego della stazione sismica. - Dalle considerazioni su riportate si può ricavare un quadro abbastanza chiaro delle possibilità offerte dalla sismologia allo studio dei vulcani, sia per quanto riguarda il tipo dei magmi sia per il tipo di attività vulcanica; possibilità che hanno attirato l'attenzione non solo degli studiosi di sismologia vulcanica ma anche quella degrli enti pubblici interessanti a scongiurare per quanto possibile distruzioni e perdite di vite umane. Le caratteristiche magmatologiche dell'Etna sono più vicine a quelle dei vulcani hawaiiani che non a quelle dei vulcani giapponesi. Tale somiglianza si riscontra anche nell'attività effusiva, che può produrre anche gravi danni alle cose pur senza essere di pericolo immediato per le popolazioni insediate sulle sue pendici.

Non è ancora possibile stabilire se anche l'attività sismica presenti analogia con quella hawaiiana ed in quale grado; sembra però ragionevole applicare inizialmente sull'Etna i metodi che più hanno dato buoni risultati sui vulcani delle Hawaii, pur tenendo nel dovuto 
conto alcune differenze già accertate dai vulcanologi, quali ad esempio la mancanza all'Etna di focolai intercrustali la cui presenza è incerta, del resto, anche altrove, la presenza di un basamento sedimentario che raggiunge lungo l'asse del vulcano quote elevate (forse $1000 \mathrm{e}$ più metri), ecc. Da questo punto di vista appare indispensabile dotare le stazioni sismografiche di postazioni clinografiche e cio rientra nei programmi dell'Istituto di Vulcanologia di Catania. Attualmente si stanno vagliando le prime registrazioni sismografiche, al fine di accertare le migliori condizioni di impiego della strumentazione di cui è dotata la stazione, con particolare riguardo ai valori più convenienti del rapporto segnale-rumore di fondo.

Superata questa prima fase preparatoria, l'attività di ricerca vera e propria sarà rivolta principalmente, nei limiti della capacità d'informazione di questa unica installazione, ai microsismi ed ai terremoti di origine vulcanica. Le caratteristiche della stazione rispondono a questi compiti. per i quali si è dovuto abbandonare un progetto precedente $\left({ }^{12}\right)$ nonostante fossero già state per esso acquistate delle apparecchiature. Questo progetto infatti prevedera la registrazione degli eventi sismici solo al di sopra di una certa intensità arbitrariamente scelta, trascurando con ciò la possibilità di informazioni derivanti dalla registrazione dei microsismi.

\section{BIBLIOGRAFIA}

(') Ritmmann A., Vulkanismus und Telktonik des Aetna. "Geologischen Rundschau ", 53, 2, 788-800, 11, 1, (Stuttgart, 1963).

${ }^{(2)}$ WILLMore P. L., The theory and design of two types of portable seismograph. "Montly notices of the Royal Astronomical Society - Geophysical Supplement ». 6, 2, 129-137, (London, 1950).

( $\left.{ }^{3}\right)$ Caloi P., Lo Surdo A., Ponte G., Agitazioni microsismiche originate da attività vulcanica, "Annali di Geofisica ", I, 1, (1948).

(4) Minaкамi T., On the investigation of explosive activities of Sakura-Jima. "Bull. Volcan. Soc. Japan ", 2, 2, 77-90, (1957).

(5) KIKUch, On the short period volcanic microtremors at Mt. Aso. "Volcanol. Soc. Japan Bull. "II, 7, 1, 1-16, (1962).

${ }^{(6)}$ Donn W. L., Microseisms. "Earth-Science Reviews ", 8, 1, 23, 213-230, (Amsterdam, 1966).

(7) Shimozuru D., Berg E., Seismological study of the Nyiragongo volcano. "Extrait du Bull. de l'Academie Royale des Sciences d'Outre-Mer, classe de Sciences techniques ", 686-712, (Bruxelles, 1961). 
(8) MinakAm T. et al., The study of eruptions and earthquakes originating from volcanoes - Part. 2. - "Volcanol. Soc. - Japan Bull. „, 2, 4, 2, 115-130 (1959) - Part. 3, 2, 4, 3, 133-151 (1960).

(') MacDonatid G. A., Eaton J. P., Hawaiian volcanoes during 1955 "Geological Survey Bulletin ", 1171, (Washington, 1963).

(10) Blot C., TAziefF H., Quelques resultats de seismologie voleanique on volcan de Tanna - Nouvelles Hebrides. "Estr. du Bull. de l'Academie Royale des Sciences d'Outre-Mer, Classe de Sciences techniques ». 270-279, (Bruxelles, 1961).

(11) OGniben L., Lineamenti idrogeologici dell'Etna (1966, in corso di stampa).

$\left({ }^{12}\right)$ Aloisio P., Progetto per l'installazione sull'Etna di una rete sismografica, collegata mediante radioonde ad un registratore centrale munito di memoria magnetica. "Atti dell'Acc. Gioenia di Sc. Nat. in Catania "VI, XV, (1963). 